\section{Prevalência de osteoporose: uma revisão crítica}

\section{Prevalence of osteoporosis: a critical review}

Paulo Frazão

Miguel Naveira

Programa de Pós-Graduação em Saúde Coletiva da Universidade Católica de Santos

Trabalho apresentado no VI Congresso Brasileiro de Epidemiologia promovido pela Associação Brasileira de Pós-Graduação em Saúde Coletiva no período de 19 a 23 de junho de 2004 na cidade de Recife-PE.

Correspondência: Universidade Católica de Santos. Programa de Pós-Graduação em Saúde Coletiva. Rua Carvalho de Mendonça 144 andar IV - CEP 11070-906 Santos, SP - Brasil

\section{Resumo}

A queda da mortalidade, seguida da redução da fecundidade e aumento da expectativa de vida, resulta no envelhecimento da população e no aumento das taxas de doenças crônico-degenerativas, entre as quais a osteoporose. Pesquisas epidemiológicas vêm sendo desenvolvidas com a finalidade de estimar sua prevalência na população empregando diferentes técnicas. O propósito dessa investigação foi revisar os estudos de prevalência de osteoporose e discutir suas implicações do ponto de vista da Saúde Coletiva. Foi realizada revisão da literatura mediante a qual foram incluídas publicações contendo estimativas de prevalência da osteoporose calculadas a partir da densidade mineral óssea aferida pela absorção de raios X de dupla energia. Os dados foram classificados segundo o ano, a região ou o país, autor, características da população investigada, e sítio esquelético. Os resultados mostraram ampla dispersão entre as taxas, com os valores variando entre $0,4 \%$ e $40,0 \%$ de acordo com o sítio pesquisado, o grupo etário, o sexo e o tipo de estudo. Nos estudos de base populacional, os valores das estimativas por ponto variaram de $7,9 \%$ a $16,0 \%$ considerando DMO no sítio femoral de mulheres com 50 e mais anos de idade. Nas áreas brasileiras mais desenvolvidas é necessário implementar projetos para conhecer sua prevalência incluindo-a na agenda dos formuladores de políticas públicas.

Palavras-chave: Políticas de Saúde. Epidemiologia. Osteoporose. Prevalência. Densidade Mineral Óssea. 


\section{Abstract}

Declining mortality, along with decreasing fecundity and increasing life expectancy, leads to an aging population and higher rates of non-transmissible chronic diseases, such as osteoporosis. There have been studies to estimate the prevalence of the event using different tools. The aim of this study was to revise osteoporosis prevalence rates and discuss the implications for Public Health. A literature review was carried out, and publications with prevalence estimates by dual energy X-ray absorptiometry were included. Data were organized according to year, country and region, author, kind of population studied, and skeletal site. The results showed a wide dispersion of rates. Values ranged between $0.4 \%$ and $40.0 \%$ depending on skeletal site, age group, gender and type of study. In population-based studies, point estimates varied from $7.9 \%$ to $16.0 \%$ considering bone mineral femoral neck densitometry in women aged $\geq 50$-years-old. It is necessary to implement projects in more developed Brazilian areas in order to describe the prevalence of osteoporosis, including it in the agenda of public policy decision makers.

Keywords: Health Policy. Epidemiology. Osteoporosis. Prevalence. Bone Mineral Density.

\section{Introdução}

Transformações ocorridas no século $\mathrm{XX}$ em certas regiões, com repercussões na urbanização, na fecundidade e no meio ambiente, têm produzido impacto na estrutura etária da população, e na distribuição da morbimortalidade, exigindo mudanças na resposta de cada sociedade aos problemas de saúde ${ }^{1}$. A queda da mortalidade (principalmente a infantil), a redução da fecundidade e o aumento da expectativa de vida resultam no envelhecimento da população e aumento das taxas de doenças crônico-degenerativas, entre as quais a osteoporose.

Esse distúrbio osteometabólico, caracterizado pela perda de massa óssea e desarranjo de sua microarquitetura, eleva a fragilidade dos ossos ${ }^{2}$. Grande parte das fraturas dela resultantes produz mudanças esqueléticas, como deformações e diminuição da estatura, com um componente doloroso importante, invalidez e até a morte $^{3}$. Estudos de metabologia mostraram que a densidade óssea aumenta até os 30 anos de idade e declina conforme uma rede complexa de fatores. Com o aumento do número de idosos, estima-se que cerca de $15 \%$ da população em todo o mundo esteja na faixa superior aos 60 anos de idade ${ }^{4}$.

A OMS estabeleceu, em 1993, consenso sobre características dos grupos etários de risco e técnicas diagnósticas de osteoporose $^{5}$. Pesquisas epidemiológicas vêm sendo desenvolvidas para estimar sua prevalência na população empregando diferentes técnicas, entre as quais a densitometria óssea medida pela absorção de raios-X de dupla energia (DXA - Dual X-Ray Absorptiometry). A densidade mineral óssea (DMO) é um importante componente de resistência do osso. O exame é considerado mais adequado e preciso, e sua medida é considerada o melhor método disponível para determinar a existência do risco e assim iniciar um tratamento preventivo ${ }^{6,7}$.

Especialistas têm preconizado que o 
diagnóstico de osteoporose e os riscos de fraturas sejam analisados pelo T-score, um valor correspondente à diferença entre a DMO média de jovens normais e a DMO do indivíduo examinado, dividido pelo desvio-padrão da média de jovens normais ${ }^{5}$.

No Brasil, o número de estudos sobre sua prevalência é pequeno, embora seja a doença osteometabólica mais comum. O propósito desta investigação foi revisar os estudos de prevalência de osteoporose empregando $D X A$ e discutir suas implicações do ponto de vista da Saúde Coletiva.

\section{Métodos}

Foi realizada revisão da literatura médica mediante consulta às principais publicações sobre o tema. O material foi identificado com auxílio das bases eletrônicas de dados bibliográficos da National Library of Medicine - MEDLINE (http:// www.ncbi.nlm.nih.gov/PubMed/); e da Literatura Latino-Americana em Ciências da Saúde - LILACS (http://www.bireme.br/). Foram empregados os descritores "epidemiologia”, "prevalência” e “osteoporose”. Considerando que levantamentos a partir de bases de dados eletrônicas contendo pesquisas científicas podem perder estudos relevantes publica$\operatorname{dos}^{8}$, e que as conclusões podem apresentar viés quando os estudos utilizados não são representativos de todas as investigações da área de pesquisa ${ }^{9}$, foi adotada como estratégia alternativa a observação minuciosa das referências contidas em artigos de revisão e nos primeiros estudos localizados a fim de identificar possíveis pesquisas que seriam perdidas se fossem utilizados apenas os meios eletrônicos.

Foram incluídas as pesquisas que continham estimativas relativas à distribuição da osteoporose na população mediante $D X A$ com base nos critérios da Organização Mundial da Saúde ${ }^{7}$ apresentados na Figura 1. Somente artigos publicados em português, espanhol, inglês, italiano e francês foram incluídos. Estudos em grupos populacionais sintomáticos, com história de fratura ou presença de fator de risco para osteoporose, editoriais, cartas e artigos indisponíveis por meio de serviço de comutação entre bibliotecas não foram considerados. Os dados epidemiológicos foram organizados conforme o lugar, o período de realização da pesquisa, as características das populações investigadas, os sítios esqueléticos examinados (coluna lombar, fêmur ou global). Na análise, a DMO femoral foi destacada em razão da medida ser considerada, para mulheres brancas, o preditor mais apropriado para risco de fratura de quadril, o tipo de fratura mais importante do ponto de vista da Saúde Coletiva ${ }^{10}$.

\section{Resultados}

Com base nos critérios adotados, foram identificados 19 estudos transversais, publicados entre 1996 e 2005. Seis estudos de base populacional e treze relativos a gru-
(1) normal
$-1$
(2) baixa massa óssea (osteopenia)
-1 a $-2,5$
(3) osteoporose
$<-2,5$
(4) osteoporose estabelecida $<-2,5$ e $(*)$

${ }^{*}$ ) pelo menos uma fratura por fragilidade óssea. Fonte: $\mathrm{WHO}^{7} 1994$.

${ }^{*}$ ) presence of one or more fragility fractures. Source: $W H O^{7} 1994$.

Figura 1 - Diferença em valores de desvio-padrão da densidade mineral óssea (DMO) observada em relação a DMO esperada para mulheres jovens saudáveis.

Figure 1 - Difference in standard deviation values of observed bone mineral density (BMD) in relation to expected BMD for young healthy women. 
pos específicos estão relacionados respectivamente nas Tabelas 1 e 2 . Considerando os estudos do primeiro tipo, pode-se observar que a faixa etária dos sujeitos examinados (homens e mulheres) variou de 20 a 80 anos de idade.

As taxas de ocorrência de osteoporose também apresentaram grande amplitude, com os valores variando entre $0,4 \%$ em mulheres em pré-menopausa até $40 \%$ naquelas com 70 anos e mais. Menor amplitude foi observada quando são consideradas apenas as estimativas obtidas a partir da DMO no sítio femoral de mulheres com 50 e mais anos de idade. Por esse critério, os valores das estimativas por ponto variaram de 7,9 a 16,0\%.

As taxas de ocorrência de osteoporose foram mais elevadas em mulheres do que em homens com idade igual ou superior a 50 anos $^{11,12,16}$.

Nenhuma diferença significativa foi observada entre as taxas de prevalência de osteoporose em coluna lombar e fêmur em mulheres com idade inferior a 50 anos.

As taxas obtidas por meio de estudos realizados em grupos específicos estão descritas na Tabela 2. Considerando as estimativas documentadas nesses estudos a partir do sítio femoral em mulheres após o período da menopausa assintomáticas recrutadas em unidades de saúde, podese observar valores entre $3,8 \%$, relatado em 473 mulheres brasileiras (em um ambulatório de Menopausa), e 22,0\%, obtido em 251 mulheres atendidas em clínica especializada na Suíça.

\section{Discussão}

Com as mudanças demográficas observadas nas regiões mais desenvolvidas do mundo, óbitos e enfermidades relacionadas às doenças crônico-degenerativas vêm

Tabela 1 - Características dos estudos transversais de base populacional sobre ocorrência de osteoporose mediante exame de densitometria óssea (DXA).

Table 1 - Features of population-based cross-sectional studies on occurrence of osteoporosis by DXA bone mineral densitometry.

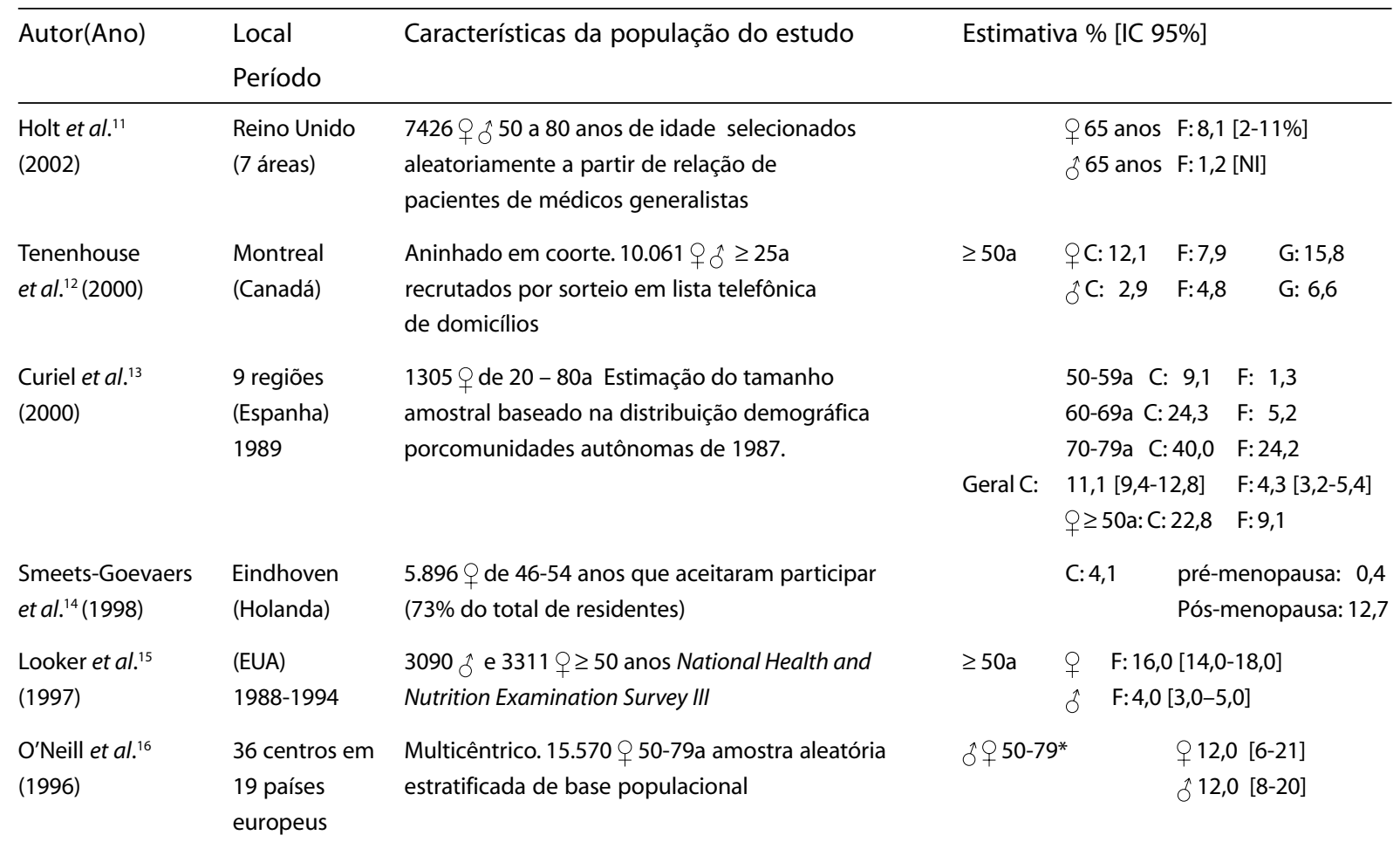

Nota: NI- não informado; Global (G): coluna lombar + fêmur; ${ }^{*}$ osteoporose estimada pela deformidade vertebral observada em exame radiográfico da coluna cervical. Note: NI- not informed; Global (G): Iumbar spine + femoral neck; * osteoporosis estimated from vertebral deformity observed in X-ray diagnosis of cervical spine. 
Tabela 2 - Características dos estudos transversais em grupos populacionais específicos sobre ocorrência de osteoporose mediante exame de densitometria óssea (DXA).

Table 2 - Features of cross-sectional studies with specific population groups on the occurrence of osteoporosis by DXA bone mineral densitometry.

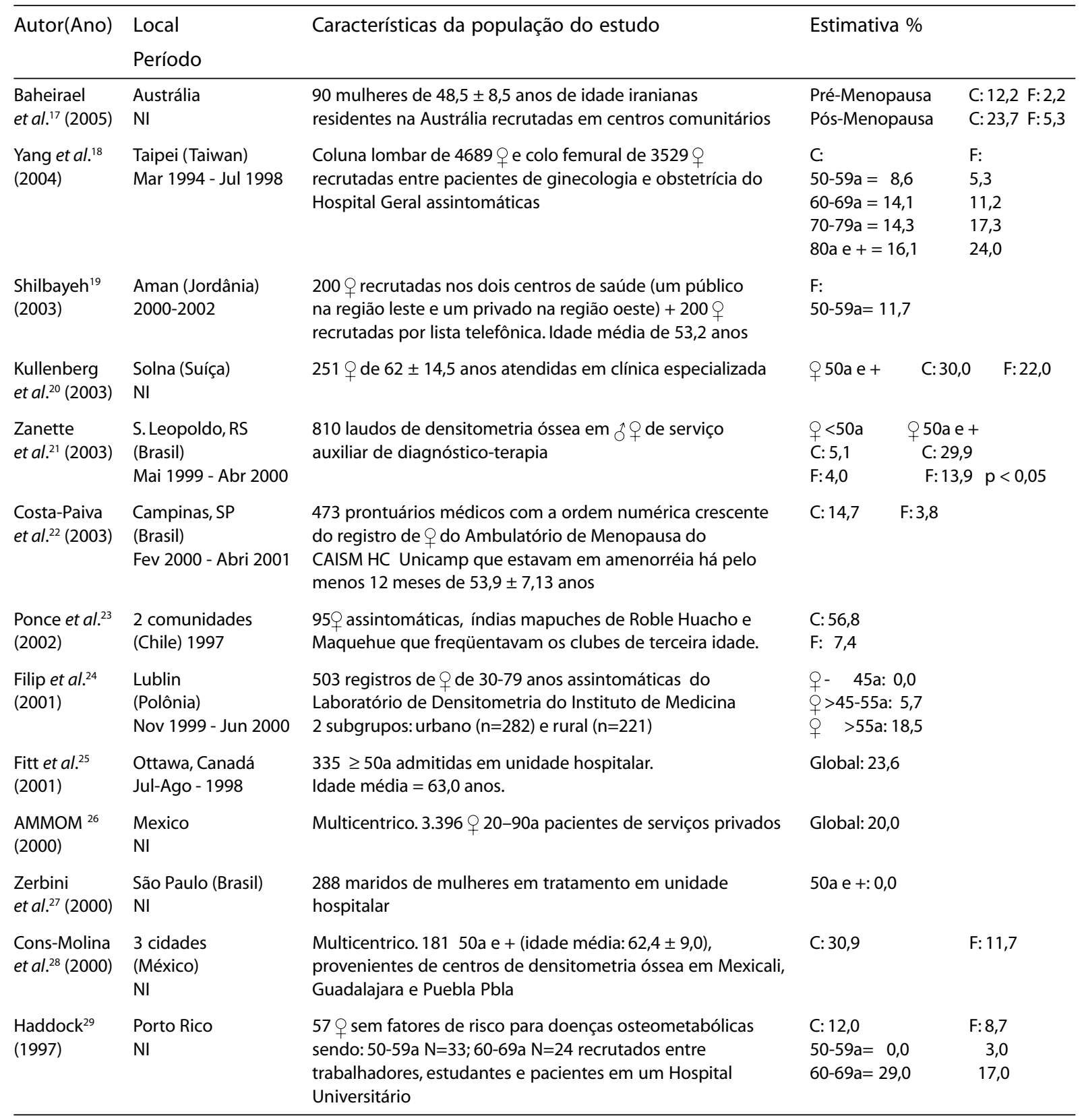

Nota: NI- não informado; Global: coluna lombar(C) + fêmur (F) / Note: Nl- not informed; Global: lumbar spine (C) + femoral neck (F)

adquirindo interesse público. Na última década do século XX, o número de estudos sobre a distribuição da osteoporose em populações cresceu, e inovações tecnológicas têm contribuído para isso.

O emprego da $D X A$ teve início em
$1987^{30}$. Tornou-se o método de escolha para a quantificação da massa óssea, sendo utilizado para diagnóstico e seguimento terapêutico. É um método não-invasivo, mais preciso na avaliação de risco de fratura, com radiação extremamente baixa e 
recomendado como o meio disponível mais adequado até o momento para avaliação de indivíduos com risco de desenvolver osteoporose ${ }^{31}$.

A adoção dos critérios estabelecidos pela OMS permite diagnosticar e tratar a doença precocemente, reduzindo a ocorrência de fraturas ${ }^{32}$. Esses critérios, originalmente definidos para mensuração em mulheres, têm sido empregados para estimar a prevalência de osteopenia e osteoporose em indivíduos acima de 50 anos de idade $^{16}$. Embora esta definição operacional possa ser criticada por alguns pesquisadores, a necessidade social de haver um padrão consistente a fim de documentar sua freqüência, e sua utilidade para subsidiar administradores de sistemas e serviços de saúde, têm justificado o seu emprego ${ }^{33}$.

A análise das características e dos resultados dos estudos de prevalência pode, entre outros aspectos, auxiliar os pesquisadores na compreensão do estado atual do assunto e na identificação das lacunas existentes no campo da produção dos conhecimentos. Além disso, subsídios podem ser oferecidos acerca da magnitude e do significado do problema em termos de Saúde Coletiva.

As taxas de ocorrência de osteoporose apresentaram grande amplitude. Características demográficas da população do estudo, entre as quais origem étnica, sexo, idade, e o tipo de sítio pesquisado - coluna lombar ou fêmur - são fatores que podem explicar essa larga variação entre os valores das estimativas.

As diferenças observadas nas taxas de osteoporose também podem ser atribuídas às características metodológicas dos estudos revisados. Entre essas características podem ser destacadas as diferentes técnicas empregadas de seleção da população. Pode-se notar desde o exame de pacientes aparentemente saudáveis recrutados aleatoriamente, mediante lista telefônica ou relação de eleitores, até a mensuração da DMO em pacientes assintomáticos para baixa massa óssea, usuários de serviços de saúde.
Além disso, parte dessas variações pode ser explicada pelos diferentes tipos de equipamento, de procedimentos de calibração e pelo padrão de referência para estimar a prevalência do problema, aspectos que têm movido pesquisadores e sociedades científicas a estabelecer uma escala de referência embasada nas características da população de cada país ${ }^{34,35}$.

Revisando as estimativas na América Latina, Morales-Torres \& GutiérrezUreña $^{36}$ relataram valores em 3 países: Colômbia, Chile e México. Os pesquisadores observaram variações semelhantes, as quais foram relacionadas ao critério de seleção das populações, incluindo aspectos étnicos que marcam a diversidade biofísica e sociocultural latino-americana.

Entre os epidemiologistas é reconhecido que, ao contrário dos estudos transversais de base populacional onde a prevalência das doenças é subestimada, em estudos de usuários de serviços de saúde, ela é de modo geral superestimada ${ }^{37}$. Com o desenvolvimento de mais estudos e a padronização de critérios de aferição e determinação das medidas de prevalência, essas diferenças poderão ser reduzidas.

Foi observado que existem dados limitados sobre as medidas ósseas no sexo masculino e nas mulheres não brancas, razão pela qual recomenda-se o desenvolvimento, não apenas de estudos transversais, mas também de estudos longitudinais em diferentes grupos étnicos para verificar a validade do critério da OMS nessas populações e determinar o risco da ocorrência de fraturas.

Nessa direção, esforços vêm sendo realizados para desenvolver técnicas de medida que controlem as discrepâncias das estimativas relacionadas ao sítio esquelético examinado, e às características demográficas, especialmente sexo e origem étnica, numa mesma população ${ }^{38}$.

As estimativas disponíveis têm mostrado que redução da massa óssea em níveis considerados de osteoporose ocorre freqüente e largamente, e causa severa deficiência e sofrimento. Investimentos em 
pesquisas voltadas para a redução dos custos do tratamento são imprescindíveis. Admitindo que existem métodos efetivos para o seu manejo e que o tratamento é aceitável, pode-se inferir que seu enfrentamento requer a intervenção da Saúde Coletiva.

Embora qualquer fratura possa ter um impacto dramático no indivíduo afetado, numa perspectiva de Saúde Coletiva as fraturas de quadril são as mais importantes ${ }^{39}$ porque são as principais fontes de morbidade associada à osteoporose e constituem causa predominante de óbito ${ }^{40,41}$.

A identificação de fatores influentes no aparecimento desse distúrbio, entre os quais idade, sexo, origem étnica, componentes genéticos, metabólicos e comportamentais, e a existência de técnicas de diagnóstico, permitem apontar grupos de alto risco, nos quais ações específicas podem conduzidas, e promover estratégias preventivas mais amplas que permitam a redução do incremento ou mesmo o controle dessa enfermidade.

No conjunto das doenças crônicas não transmissíveis, os aspectos mencionados e os custos crescentes com a assistência à saúde, têm levado a considerar a osteoporose um problema relevante para aqueles que se ocupam da Saúde Pública mun$\operatorname{dial}^{7,42,43}$.

Na Europa, especialistas têm chamado a atenção e formulado recomendações (Figura 2) para enfrentar o problema ${ }^{44}$. No Brasil, algumas dessas recomendações podem ser consideradas na agenda das autoridades sanitárias responsáveis pela gestão das ações e serviços de saúde nas regiões mais desenvolvidas, onde a população apresenta características semelhantes.

Nas regiões mais desenvolvidas do nosso país, onde existem áreas intramunicipais com taxas de residentes com 60 e

1. A Comissão Européia e os governos dos 15 Estados-membros, em especial os responsáveis pelos processos decisórios e as autoridades nacionais, devem apontar explicitamente a prevenção da osteoporose como uma das grandes áreas dos cuidados de saúde e criar campanhas de conscientização. A prevenção da osteoporose deve ter elevada prioridade na promoção da saúde, na educação para a saúde e na formação dos profissionais de assistência à saúde.

2. Criar sistemas coordenados de monitoração das taxas de fratura, em cada país e na Comunidade Européia.

3. Assegurar a coordenação dos sistemas nacionais no âmbito da UE, e planejar mais eficazmente a repartição de recursos e divisão de tarefas com vistas a uma resposta mais adequada ao problema.

4. Integrar, implementar, e, se adequado, desenvolver políticas de educação do público em geral e dos profissionais da saúde sobre o cálcio e a vitamina D na nutrição em todas as fases da vida.

5. Permitir o acesso das pessoas de alto risco ao exame de densitometria óssea e torná-las reembolsáveis.

6. Elaborar e coordenar linhas de orientação sobre os critérios relativos a estratégias de tratamento normalizadas. Todos os tratamentos aprovados devem ser reembolsados.

7. Oferecer apoio às associações nacionais científicas e de doentes na postulação de uma resposta mais eficaz ao problema por parte das autoridades.

8. Financiar investigação mais pormenorizada em áreas fundamentais, com a finalidade de implementar melhores estratégias preventivas da osteoporose.

Fonte: International Osteoporosis Foundation. European Commission ${ }^{44} 1998$.

Source: International Osteoporosis Foundation. European Commission ${ }^{44} 1998$.

Figura 2 - Recomendações de Especialistas Europeus para Estados-membros. Figure 2 - Policy Recommendations of European Experts for Member States. 
mais anos de idade ao redor de $20 \%$, e os índices de envelhecimento são crescentes, a presença desse distúrbio na população idosa pode elevar o risco de fraturas com conseqüente perda funcional e aumento da utilização dos serviços de saúde. Pesquisadores têm chamado atenção das autoridades sanitárias ${ }^{45,46}$ para as implicações sociais e econômicas do problema na população brasileira ${ }^{47,48}$. Por essas, entre outras razões, é necessário implementar nessas áreas projetos para descrever e monitorar sua distribuição na população, incluindo-a tanto na agenda dos pesquisadores quanto naquela dos formuladores de políticas públicas.

\section{Referências}

1. Laurenti R. Transição demográfica e transição epidemiológica. In: Anais do I Congresso Brasileiro de Epidemiologia. Campinas: Brasil; 1990.

2. Consensus Development Conference. Diagnosis, prophylaxis, and treatment of osteoporosis. Am J Med 1993; 94: 646-50.

3. Riggs BL. Pathogenesis of osteoporosis. Am J Obstet Gynecol 1987; 156(5): 1342.

4. Albuquerque SMRL. Envelhecimento ativo: desafio dos serviços de saúde para a melhoria da qualidade de vida dos idosos. [tese de doutorado] São Paulo: Universidade de São Paulo; 2005.

5. World Health Organization. Assessment of fracture risk and application to screening for postmenopausal osteoporosis. Washington, DC: Technical Report Series 843; 1994. p.1-129.

6. National Institute of Health. Consensus Development Panel on Osteoporosis prevention, Diagnosis and Therapy. Osteoporosis: prevention, diagnosis and therapy. JAm Med Assoc 2001; 285: 785.

7. Meunier PJ, Delmas PD, Eastell R, McClung MR, Papapoulos S, Rizzoli R, et al. Diagnosis and management of osteoporosis in postmenopausal women: clinical guidelines. International Committee for Osteoporosis Clinical Guidelines. Clin Ther 1999; 21(6): 1025-44.

8. Dickersin K, Hewitt P, Mutch L, Chalmers I, Chalmers TC. Perusing the literature: comparison of MEDLINE searching with a perinatal clinical trials database. Control Clin Trials 1985; 6: 306-17.

9. Lavalley M. A consumer's guide to meta-analysis. Arthritis Care Res 1997; 10(3): 208-13.

10. Marshall D, Johnell O, Wedel H. Meta-analysis of how well measures of bone mineral density predict occurrence of osteoporotic fractures. BMJ 1996; 312: 1254-59.

11. Holt G, Khaw KT, Reid DM, Compston JE, Bhalla A, Woolf AD, Crabtree NJ, Dalzell N, Wardley-Smith B, Lunt M, Reeve J. Prevalence of osteoporotic bone mineral density at the hip in Britain differs substantially from the US over 50 years of age: implications for clinical densitometry. British Journal of Radiology 2002; 75: 736-42.
12. Tenenhouse A, Joseph L, Kreiger N, Poliquin S, Murray TM, Blondeau L, Berger C, Hanley DA, Prior JC; CaMos Research Group. Canadian Multicentre Osteoporosis Study. Estimation of the prevalence of low bone density in Canadian women and men using a population-specific DXA reference standard: the Canadian Multicentre Osteoporosis Study (CaMos). Osteoporos Int 2000; 11(10): 897-904.

13. Curiel M D, Garcia J J, Carrasco J L, Honorato J, Pérez Cano R, Rapado A, Álvarez Sanz C. Prevalencia de osteoporosis determinada por densitometría en la población femenina española. Med Clin 2001; 116: 86-88.

14. Smeets-Goevaers CG, Lesusink GL, Papapoulos SE, Maartens LW, Keyzer JJ,Weerdenburg JP, Beijers LM, Zwinderman AH, Knottnerus JA, Pols HA, Pop VJ. The prevalence of low bone mineral density in Dutch perimenopausal women: the Eindhoven perimenopausal osteoporosis study. Osteoporos Int 1998; 8(5): 404-9.

15. Looker A C, Orwoll E S, Johnston Jr. C C, Lindsay R L, Walner H W, Dunn W L, Calvo MS, Harris T B, Heyse S P. Prevalence of low femoral bone density in older US adults from NHANES III. J Bone Miner Res 1997, 12 (11): 17611768.

16. O'Neill TW, Varlow J, Cooper C et al. Differences in vertebral deformity indices between European populations. J Bone Miner Res 1993; 8 (Supl 1):S149.

17. Baheirael A., Pocock NA, Eisman JA, Nguyen ND, Nguyen TV. Bone mineral density, body mass index and cigarette smoking among Iranian women: implications for prevention BMC Musculoskeletal Disorders 2005, 6:34.

18. Yang TS, Chen YR, Chen YJ, Chang CY, Ng HT. Osteoporosis: prevalence in Taiwanese women. Osteoporosis International 2004; 15(4): 345-7.

19. Shilbayeh S. Prevalence of osteoporosis and its reproductive risk factors among Jordanian women: a cross-sectional study. Osteoporosis International 2003; 14(11): 929-40.

20. Kullenberg R, Falch JA. Prevalence of osteoporosis using bone mineral measurements at the calcaneus by dual Xray and laser (DXL). Osteoporos Int 2003;14(10):823-7. 
21. Zanette E, Stringari FF, Machado F, Marroni BJ, Ng DPK, Canani LH. Avaliação do diagnóstico densitométrico de osteoporose/osteopenia conforme o sítio ósseo. Arq Bras Endocrinol Metab 2003; 47(1): 30-6.

22. Costa-Paiva L, Horovitz A P, Santos A O, FonsechiCarvasan G A, Pinto-Neto A M. Prevalência de osteoporose em mulheres na pós-menopausa e associação com fatores clínicos e reprodutivos. Rev Bras Ginecol Obstet 2003; 25(7) 507-12.

23. Ponce L, Larenas G, Riedemann P. Alta prevalencia de osteoporosis en mujeres mapuches postmenopáusicas asintomáticas. Rev Méd Chile 2002; 130: 1365-72.

24. Filip RS, Zagórski J. Bone mineral density and osteoporosis in rural and urban women. Epidemiological study of the Lublin region (Eastern Poland). Ann Agric Environ Med 2001; 8: 221-6.

25. Fitt N S, Mitchell S L, Cranney A, Gulenchyn K, Huang M, Tugwell P. Influence of bone densitometry results on the treatment of osteoporosis. Can Med Assoc J2001; 20:164 (6): 777-81.

26. Asociacion Mexicana de Metabolismo oseo y Mineral. Consenso Mexicano de Osteoporosis. Juriquilla, México: oct 2000. URL: http:/ /www. osteoporosis-center.com/ ostcenter.com/c014.htm capturado em março de 2004.

27. Zerbini CAF, Latorre MRDO, Jaime PC, Tanaka T, Pippa MGB. Bone mineral density in Brazilian men 50 years and older. Braz J Med Biol Res 2000; 33(12): 1429-35.

28. Cons-Molina F, Hinojosa MD, Rodríguez MPP. Análisis de Eficiencia de las Estrategias para Identificar Mujeres Postmenopáusicas con Osteoporosis en México. URL: http:// www.osteoporosis-center.com/ostcenter.com/ c006-6.htm capturado em março de 2004.

29. Haddock L. Prevalence of osteopenia and osteoporosis in a normal female Puerto Rican population. $P R$ Health Sci $J$ 1997; 16(3): 241-4.

30. Lang P, Steiger P, Faulkner K, Gluer C, Genant H K. Osteoporosis. Current techniques and recent developments in quantitative bone densitometry. Radiol Clin North Am 1991; 29(1): 49-76.

31. Seeley D G, Browner W S, Nevitt M C. Which fractures are associated with low appendicular bone mass in elderly women? Ann Intern Med 1991; 115: 837-42.

32. Kanis JA, Melton III LJ, Cristiansen C, Johnston CC, Khaltaev N. The diagnosis of osteoporosis. J Bone Miner Res. 1994, 8: 1137-41.

33. Melton III, LJ. The Prevalence of Osteoporosis. J Bone and Mineral Res 1997; 12(11): 1769-71. [Editorial]

34. Binkley N, Kiebzak GM, Lewiechi EM, Krueger D, Gangnon RE, Miller PD, Shepherd JA, Drezner MK. Recalculation of the NHANES Database SD Improves TScore Agreement and Reduces Osteoporosis Prevalence. Journal of Bone and Mineral Research 2005; 20(2): 195201.
35. Henry MJ, Pasco JA, Pocock NA, Nicholson GC, Kotowics MA. Reference ranges for bone densitometers adopted Austrália-wide: Geelong Osteoporosis Study. Australasian Radiology 2004; 48: 473-5.

36. Morales-Torres J, Gutiérrez-Ureña S. The burden of osteoporosis in Latin America. Osteoporos Int 2004; 15: 625-32.

37. Pereira MG. Epidemiologia: teoria e prática. Rio de Janeiro: Guanabara Koogan; 1995.

38. Melton LJ III. The prevalence of osteoporosis: gender and racial comparison. Calcif Tissue Int 2001; 69: 179-81.

39. Melton LJ III. Who has osteoporosis? A conflict between clinical and public health perspectives. J Bone Miner Res 2000; 15 (12): 2309-14.

40. Cooper C, Atkinson EJ, Jacobsen SJ, O'Fallon WM, Melton LJ III. Population-based study of survival after osteoporotic fractures. Am J Epidemiol 1993; 137: 1001-5.

41. Center JR, Nguyen TV, Schneider D, Sambrook PN, Eisman JA. Mortality after all major types of osteoporotic fracture in men and women: an observational study. Lancet 1999; 353: 878-82.

42. Johnell O, Gullberg B, Allander E. MEDOS Study Group. The apparent incidence of hip fracture in Europe: a study of National Register Sources. Osteoporosis Int 1992; 2: 298-302.

43. Vellas B, Gillette-Guyonnet S, Nourhashémi F, Rolland Y, Lauque S, Ousset PJ, Moulias S, Andrieu S, Fontan B, Adone D, Lafont C, Baumgarten R, Garry P, Albarède JL. Chutes, fragilité et osteóporose chez la personne âgée: un problème de santé publique. Rev Méd Interne 2000; 21: 608-13.

44. International Osteoporosis Foundation. European Commission. Report on Osteoporosis in the European Community: Action for Prevention. Luxembourg: Office for Official Publications of the European Communities, 1998. Obtido em URL: http://www. osteofound.org/ advocacy_policy/recommendations.html

45. National Consensus Proposal. Basic diagnosis and therapeutic elements for a "National Consensus Proposal”. São Paulo Medical Journal 1995; 113 (Supl.): 718.

46. Pinto-Neto AM, Soares A, Urbanetz AA, Souza ACA, Ferrari AEM, Amaral B et al. Consenso Brasileiro de Osteoporose - 2002. Revista Brasileira de Reumatologia 2002; 42(6): 343-54.

47. Carneiro RA. Repercussões socioeconômicas da osteoporose no Brasil: estimativas de custos. Arq Bras Med 1988; 62(1): 65-9.

48. Lautert L, Almeida MA, Araújo VG, Francisco CMC. Osteoporose: a epidemia silenciosa que deve se tornar pública Rev Bras Enfermagem 1995; 48(2):161-7.

Recebido em: 30/08/05 Versão reformulada reapresentada em: 29/05/06 Aprovado em: 30/05/06 